\title{
Implementing an apparent-horizon finder in three dimensions
}

\author{
Thomas W. Baumgarte, ${ }^{*}$ Gregory B. Cook, and Mark A. Scheel \\ Center for Radiophysics and Space Research, Cornell University, Ithaca, New York 14853 \\ Stuart L. Shapiro ${ }^{\dagger}$ \\ Center for Astrophysics and Relativity, 326 Siena Drive, Ithaca, New York 14850 \\ Saul A. Teukolsky \\ Center for Radiophysics and Space Research, Cornell University, Ithaca, New York 14853
}

(Received 6 June 1996)

\begin{abstract}
Locating apparent horizons is not only important for a complete understanding of numerically generated spacetimes, but it may also be a crucial component of the technique for evolving black-hole spacetimes accurately. A scheme proposed by Libson, Massó, Seidel, and Suen, based on expanding the location of the apparent horizon in terms of symmetric trace-free tensors, seems very promising for use with threedimensional numerical data sets. In this paper, we generalize this scheme and perform a number of code tests to fully calibrate its behavior in black-hole spacetimes similar to those we expect to encounter in solving the binary black-hole coalescence problem. An important aspect of the generalization is that we can compute the symmetric trace-free tensor expansion to any order. This enables us to determine how far we must carry the expansion to achieve results of a desired accuracy. To accomplish this generalization, we describe a new and very convenient set of recurrence relations which apply to symmetric trace-free tensors.

[S0556-2821(96)03720-4]
\end{abstract}

PACS number(s): 04.20.Cv, 02.60.Cb, 02.70.Rw, 04.25.Dm

\section{INTRODUCTION}

A major goal of numerical relativity is to simulate the coalescence of an orbiting pair of black holes. In studying such systems, we will be interested in determining many quantities: the energy and momentum radiated, the associated wave forms, the total angular momentum of the system, etc. In addition to these common physical quantities, we will also want to understand the causal structure of the spacetime. Not only will this give us a more complete picture of the dynamics, but it also seems that tracking the causal structure may prove to be a crucial step in successfully evolving black-hole spacetimes [1]. Knowing which events are inside black holes may allow them to be excised from the computational domain, thereby avoiding numerical difficulties that have plagued black hole evolutions. Ideally, we would like to be able to track all of the event horizons in a given spacetime. However, this is not possible during the evolution: event horizons can only be reconstructed after the evolution is complete. Instead, apparent horizons can be located on each individual spacelike hypersurface during an evolution.

\footnotetext{
*Present address: Department of Physics, University of Illinois at Urbana-Champaign, Urbana, IL 61801. Also at National Center for Superconductivity Applications, University of Illinois at UrbanaChampaign, Urbana, IL 61801.

†Present address: Department of Physics, University of Illinois at Urbana-Champaign, Urbana, IL 61801. Also at Department of Astronomy and National Center for Supercomputing Applications, University of Illinois at Urbana-Champaign, Urbana, IL 61801.

${ }^{\ddagger}$ Also at the Departments of Physics and Astronomy, Cornell University.
}

Since apparent horizons must lie inside event horizons and asymptote towards them as the system settles down, they provide much of the desired causal information. They can also be used to define regions that can be excised from a computation.

Various methods exist for locating apparent horizons. In practice, one searches for marginally outer-trapped surfaces (MOTS's) [2]. The apparent horizon is the outermost such surface. For axisymmetric problems, shooting methods [3-6] have been the most widely used for locating apparent horizons, although decomposition into orthogonal polynomials [7], the solution of elliptic boundary-value problems [8,9], and the use of curvature flows [10] have also been used. Unfortunately, shooting methods do not generalize to threedimensional spatial slices. The first general apparent-horizon finders were based on a spherical-harmonic decomposition of the MOTS [11-13]. In this approach, each coefficient in the spherical-harmonic expansion is determined, iteratively, by performing an integral over a complicated function that characterizes the MOTS. The MOTS equation can also be posed as an elliptic equation for a function that parametrically specifies the location of the MOTS [14]. Curvature flow methods are also certainly applicable in the general case of a three-dimensional spatial hypersurface.

Recently, a variant of the spherical-harmonic decomposition method has been proposed by Libson et al. [15]. This approach is conceptually appealing, having two particularly nice features. First, the coefficients in the spherical-harmonic expansion are determined by a minimization procedure, eliminating the need to perform surface integrals. Second, Libson et al. have proposed the use of symmetric trace-free (STF) tensors for parametrically representing the MOTS. This latter feature is particularly appealing when Cartesian 
coordinates are used on the three-dimensional hypersurface.

In this paper, we will review the method proposed by Libson et al. and describe how we generalize the method by extending the expansion in STF tensors to arbitrary order. Because one is always using a truncated expansion, it is important to understand clearly the behavior of the apparenthorizon finder when the maximum order of the expansion is varied. As we will see, the number of points where the MOTS is determined will also affect the behavior of the apparent-horizon finder. We have examined both of these effects in detail.

The paper is organized as follows. In Sec. II we outline the method and basic equations, and in Sec. III we explain our numerical implementation. In Sec. IV we carefully discuss results from various test calculations, and in Sec. V we briefly summarize the most important results. All technical details are provided in the appendices. Appendix A contains a number of useful equations relating to STF tensors. Appendix B describes the storage for arbitrary rank tensors. In Appendix $\mathrm{C}$ we derive recurrence relations for STF tensors. Finally, in Appendix D we derive an expression for the area element on the apparent horizon.

\section{METHOD AND BASIC EQUATIONS}

A MOTS is a closed two-surface embedded in a threedimensional spatial hypersurface and, therefore, can be defined as a level surface of some scalar function $\tau$. If we use Cartesian coordinates on the hypersurface then we can parametrically define the level surface as

$$
\tau(x, y, z)=\sqrt{\delta_{i j}\left(x^{i}-C^{i}\right)\left(x^{j}-C^{j}\right)}-f(\theta, \phi)=0 .
$$

Here, $x^{i}$ are Cartesian coordinates, $C^{i}$ is a location inside the $\tau=0$ surface, and $\theta$ and $\phi$ are polar coordinates centered on $C^{i}$. The function $f(\theta, \phi)$ then measures the coordinate distance between $C^{i}$ and the $\tau=0$ surface in the direction $(\theta, \phi)$. The outward-pointing unit normal on the $\tau=0$ surface is

$$
S_{i}=\lambda \partial_{i} \tau
$$

where $\lambda$ is the normalization factor,

$$
\lambda \equiv\left[g^{i j}\left(\partial_{i} \tau\right)\left(\partial_{j} \tau\right)\right]^{-1 / 2},
$$

and $g_{i j}$ is the metric on the spatial hypersurface. The expansion $\Theta$ of an outgoing null bundle can now be written

$$
\Theta=D_{i} S^{i}+K_{i j} S^{i} S^{j}-K_{i}^{i},
$$

where $K_{i j}$ is the extrinsic curvature and $D_{i}$ is the covariant derivative operator associated with $g_{i j}$. Note that the term $D_{i} S^{i}$ involves both first and second derivatives of $\tau$ and hence of $f(\theta, \phi)$. Writing these out explicitly yields

$$
\Theta=\left(g^{i j}-S^{i} S^{j}\right)\left(\frac{\lambda}{f}\left(\delta_{i j}-n_{i} n_{j}\right)-\lambda \partial_{i} \partial_{j} f-S_{k} \Gamma_{i j}^{k}-K_{i j}\right) .
$$

Here, $\delta_{i j}$ is the Kronecker delta, $n^{i}$ is the unit vector in the $(\theta, \phi)$ direction, and $\Gamma_{i j}^{k}$ are the connection coefficients associated with $g_{i j}$. One must be careful in deriving this equa- tion since there are effectively two metrics being used: the spatial metric $g_{i j}$ and the Kronecker delta $\delta_{i j}$ used in computing coordinate distances. In particular, we note that

$$
\begin{gathered}
n^{i}=\frac{x^{i}-C^{i}}{\sqrt{\delta_{j k}\left(x^{j}-C^{j}\right)\left(x^{k}-C^{k}\right)}}, \\
n_{i} \equiv \delta_{i j} n^{j} \quad\left(\delta_{i j} n^{i} n^{j}=1\right), \\
S_{i}=\lambda\left(n_{i}-\partial_{i} f\right), \\
S^{i} \equiv g^{i j} S_{j} \quad\left(g_{i j} S^{i} S^{j}=1\right) .
\end{gathered}
$$

Our goal is now to find a function $f(\theta, \phi)$ such that the $\tau=0$ surface is a MOTS, i.e., that the expansion (5) vanishes on that surface. In practice, instead of making the expansion vanish, we can evaluate it at a number $N_{\Theta}$ of points on the surface $\tau=0$ and look for a function $f$ such that

$$
\mathcal{S}\left(N_{\Theta}\right) \equiv \sum_{\alpha=1}^{N_{\Theta}} W_{\alpha} \Theta_{\alpha}^{2}
$$

vanishes. If Eq. (10) vanishes for arbitrary weights $W_{\alpha}$, then in the limit $N_{\Theta} \rightarrow \infty$ (so as to completely cover the surface) we are guaranteed of having located a MOTS.

Our strategy will be to expand $f(\theta, \phi)$ in terms of multipole moments and to search for a minimum in $\mathcal{S}$. The sum (10) then depends on the corresponding expansion coefficients, which can be varied until the sum assumes a minimum. If this minimum comes arbitrarily close to zero, an apparent horizon has been found. Thus, the problem has been reduced to a multi-dimensional minimization, for which standard methods can be used.

An obvious choice of basis functions for the expansion of $f(\theta, \phi)$ are the spherical harmonics,

$$
f(\theta, \phi)=\sum_{l=0}^{L} \sum_{m=-l}^{l} F^{l m} Y^{l m}(\theta, \phi),
$$

where the expansion is truncated at order $L$. However, since we have to take up to second derivatives with respect to Cartesian coordinates, an expansion in terms of STF tensors

$$
f(\theta, \phi)=\sum_{l=0}^{L} \mathcal{F}_{K_{l}} N_{K_{l}}
$$

turns out to be a better choice. In the following we adopt the notation of [16], where additional details of this formalism can be found. In particular, repeated indices will always be summed over. The subscript $K_{l}$ denotes a multi-index of length $l$, and $N_{K_{l}}$ is the vector product of $l$ unit vectors $n_{i}$ :

$$
N_{K_{l}}=n_{k_{1}} n_{k_{2}} \cdots n_{k_{l}}
$$

In Eq. (12), these are contracted with the STF tensors $\mathcal{F}_{K_{l}}$ (of rank $l$ ). These are the location-independent expansion coefficients equivalent to the $F^{l m}$ in Eq. (11). Note that a STF tensor of rank $l$ has $(2 l+1)$-independent components, just like the spherical harmonics. The relationship between Eqs. (11) and (12) can be seen even more clearly by choosing the 
$\mathcal{Y}_{K_{l}}^{y^{m}}$ basis for the STF tensors as defined in Ref. [16] (see Appendix A). In terms of these, $\mathcal{F}_{K_{l}}$ can be written

$$
\mathcal{F}_{K_{l}}=\sum_{m=-l}^{l} F^{l m} \mathcal{Y}_{K_{l}}^{l m}
$$

where the $F^{l m}$ are the same as in Eq. (11). The $\mathcal{Y}_{K_{l}}^{l m}$ also provide a relation between the spherical harmonics and the $N_{K_{l}}$ :

$$
Y^{l m}(\theta, \phi)=\mathcal{Y}_{K_{l}}^{l m} N_{K_{l}}(\theta, \phi) .
$$

Inserting this into Eq. (11) and using Eq. (14) immediately yields Eq. (12).

Note that because the coefficients $\mathcal{F}_{K_{l}}$ are location independent, the derivatives of $f$ can be calculated from the derivatives of $N_{K_{l}}$,

$$
\partial_{i} f(\theta, \phi)=\sum_{l=0}^{L} \mathcal{F}_{K_{l}} \partial_{i} N_{K_{l}},
$$

and similarly for second derivatives.

\section{NUMERICAL IMPLEMENTATION}

Our code is designed in such a way that it can find a MOTS to arbitrary order $L$ in the multipole expansion. On input we, therefore, have to specify the order $L$. Also, we have to specify the number of points $N_{\Theta}$ on the surface at which the expansion (5) (and, of course, the sum for $\mathcal{S}$ ) are evaluated. These points must be distributed somehow over the surface. Currently, they are distributed equally in $\phi$ and $\cos \theta$ on the unit sphere, but different choices could easily be made. Results for different values of $L$ and $N_{\Theta}$ will be presented in the next section.

Next, the tensors $N_{K_{l}}$, their first and second derivatives, as well as the basis STF tensors $\mathcal{Y}_{K_{l}}^{l m}$, have to be initialized. The latter are independent of location, so that we need to calculate them only once. The $N_{K_{l}}$ do, however, depend on the direction $(\theta, \phi)$, and, therefore, have to be calculated once for every point on the surface. In the code, we define arrays of length $N_{\Theta}$ to store $N_{K_{l}}$ and its derivatives.

Since all of the STF tensors are completely symmetric, we can store the independent components in a very elegant and efficient way. This is explained in detail in Appendix B. In Appendix $\mathrm{C}$ we present recurrence relations that allow for a very efficient initialization of these objects.

The MOTS search is started with a set of trial expansion coefficients $F^{l m}$. These are then contracted with the basis STF tensors $\mathcal{Y}_{K_{l}}^{l m}$, which yields the $\mathcal{F}_{K_{l}}$. Since these quantities are independent of location, this needs to be done only once per iteration step.

The $\mathcal{F}_{K_{l}}$ are then contracted with $N_{K_{l}}$ and its derivatives to find $f, \partial_{i} f$, and $\partial_{i} \partial_{j} f$ for each direction $(\theta, \phi)$. Once $f$ is known, we can construct the coordinate location

$$
x^{i}=f n^{i}+C^{i}
$$

of the trial surface $(\tau=0)$ at each of the $N_{\Theta}$ points on the surface. For each point, we read in $g_{i j}, K_{i j}$, and $\Gamma_{i j}^{k}$. These can either be numerically evolved quantities or, for the test purposes in this paper, analytical values. Equation (5) now yields the expansion $\Theta$ for this location on the trial surface. Repeating these steps for every point $N_{\Theta}$ we can finally construct the sum $\mathcal{S}$. Currently, we choose the weights $W_{\alpha}$ in Eq. (10) based on the proper area element (D13) defined in Appendix D. Thus, equation Eq. (10) is an approximation to the mean square of the expansion:

$$
\mathcal{S}=\oint \Theta^{2} d^{2} \sigma
$$

Any multi-dimensional minimization routine can now be used to vary the $F^{l m}$ until $\mathcal{S}$ has assumed a minimum. So far, we have found best results with Powell's method [17], although it is likely that a method that uses derivatives with respect to the expansion coefficients $F^{l m}$ will be significantly faster, especially when the initial guess is already close to the final answer. We hope to explore this in the future.

Once a minimum has been found, we shift the center of the black hole $C^{i}$ according to the dipole moment (i.e., we choose $C^{i}$ so that the $l=1$ moments vanish). We then repeat the MOTS search until the $l=1$ moments stay below a predetermined maximum value. This procedure enables us to locate apparent horizons even when the initial guess is very poor (see next section) and should allow us to follow black holes that move through a numerical grid.

\section{TESTS}

\section{A. Schwarzschild}

An obvious test for the apparent-horizon finder is the Schwarzschild spacetime. Since the MOTS is spherically symmetric, it can be described with the monopole term alone.

This test strongly demonstrated how well the shifting of the center $C^{i}$ according to the $l=1$ moments works. The code was able to locate the MOTS accurately even when the initial guess was completely disjoint from the true horizon. The code worked equally well when we located the black hole away from the origin of the coordinate system. In all cases the sum $\mathcal{S}$, as well as all expansion coefficients $F^{l m}$ with $l>0$, vanished to whatever tolerance we specified.

\section{B. Two black holes}

A spacetime containing two black holes has multiple MOTS's, some of which can be highly distorted. Such a spacetime provides a much stronger test for the apparenthorizon finder.

A metric for two time-symmetric black holes can be written in the conformally flat form

$$
d s^{2}=\psi^{4}\left(d x^{2}+d y^{2}+d z^{2}\right),
$$

where the conformal factor $\psi$ is given by

$$
\psi=1+\frac{M}{2 r_{1}}+\frac{M}{2 r_{2}}
$$




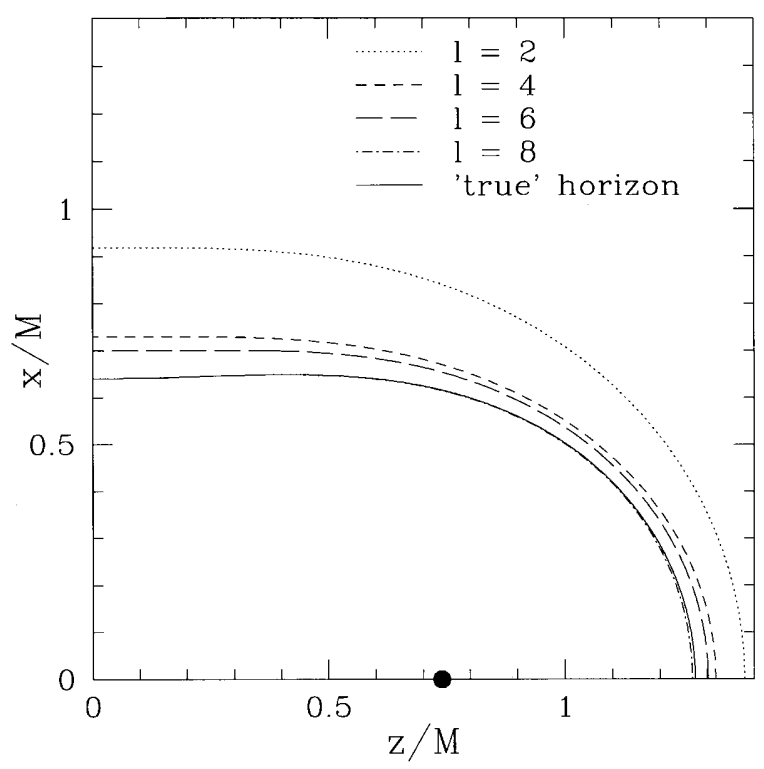

FIG. 1. The estimated location of the MOTS's found with expansions taken to order $L=2,4,6$, and 8 for $z_{0}=0.74 M$ (using $N_{\Theta}=25 \times 11$ points). The "true location" was found independently by solving a set of coupled differential equations (see Ref. [6]). The dot marks the coordinate center of one of the two black holes.

and $r_{1}$ and $r_{2}$ are

$$
\begin{aligned}
& r_{1}=\left[x^{2}+y^{2}+\left(z+z_{0}\right)^{2}\right]^{1 / 2}, \\
& r_{2}=\left[x^{2}+y^{2}+\left(z-z_{0}\right)^{2}\right]^{1 / 2} .
\end{aligned}
$$

Here, $M$ is the mass of the individual black holes, and $z_{0}$ is their coordinate distance from the origin of the coordinate system.

Note that the singularities in Eq. (20) can be removed by adding matter sources (see Ref. [6]). Since this is advantageous in a numerical application and does not change the external metric we have implemented this form of the equations.

The causal structure of this spacetime has been investigated in detail by Bishop [4]. The MOTS can be found by using a shooting method to solve a set of coupled differential equations to high accuracy. This provides us with a solution that we can check the STF-based apparent-horizon finder against. In the following we will refer to these solutions as the "true horizons."

In general, there will be MOTS's around the individual holes. If the holes are close enough, a pair of encompassing MOTS's will also appear (see Ref. [4] for a careful discussion). According to Cadež [3] these encompassing MOTS's first appear at a separation of $z_{0}=0.765 M$. For separations close to the critical separation (i.e., $z_{0} \lesssim 0.765 M$ ) they will be strongly distorted.

Note that this situation is quite similar to what we expect in a binary black-hole evolution. Having that application in mind, it provides a strong test for our apparent-horizon finder and it can help us to decide to which order $L$ we need to expand and at how many points $N_{\Theta}$ we need to evaluate the expansion $\Theta$ in order to accurately locate the encompassing MOTS's.
In Fig. 1 we plot the estimated location of the MOTS based on expansions to order $L=2,4,6$, and 8 for the case $z_{0}=0.74 M$. (By symmetry, only even $L$ can contribute since $C^{i} \rightarrow 0$.) The "true horizon" is fairly distorted, causing the lower-order expansions to perform very poorly.

Note that all the lower-order expansions find a location for the MOTS outside of the true horizon. This behavior could have severe consequences in numerical evolution codes that use "apparent horizon boundary conditions" [1] and that ignore the causally disconnected region inside a MOTS. If we were to use one of the lower-order MOTS solutions for this purpose we could be ignoring a region that is not causally disconnected.

This test clearly demonstrates that high-order expansion is absolutely necessary for the detection of highly distorted horizons. On the other hand, it also demonstrates that highorder expansion is very expensive: increasing the order of the expansion by 2 increases the CPU time by roughly a factor of 3, ranging from several seconds for $L=2$ to several minutes for $L=8$ (on a serial computer). Details will depend on the particular numerical implementation as well as parameters associated with a given minimization routine. However, since we are searching for minima in an $(L+1)^{2}$-dimensional space [see Eq. (B9)], the required CPU time will always be a steep function of $L$.

Another important factor for both the accuracy and the CPU time is the number of points $N_{\Theta}$ at which the expansion $\Theta$ is evaluated. In Fig. 2 we show results for $z_{0}=0.6$, using different numbers of points $N_{\Theta}=n_{\theta} \times n_{\phi}$, where $n_{\theta}$ is the number of points in the $\theta$ direction and $n_{\phi}$ in the $\phi$ direction.

Since the $F^{l m}$ up to order $L$ have $(L+1)^{2}$-independent components, we will need at least $(L+1)^{2}$ points. From Fig. 2 it is obvious that $8 \times 8$ points are not enough for an expansion to order $L=8$ : the result is worse than that for a lowerorder expansion. However, it can also be seen that increasing the number of points beyond this minimum can drastically increase the accuracy.

As a next test we plot in Fig. 3 the integral (18) as a function of $z_{0}$ for different expansion orders. For all these calculations we start with an initial guess close to where we expect a common MOTS. For $z_{0}<0.765 M$, when the two black holes have a common MOTS, we would, therefore, expect this sum to vanish, if we could resolve the MOTS arbitrarily well. For values of $z_{0}$ larger than $0.765 M$ the minimization routine will find a nonzero minimum; however, we expect these minima to go to zero as we approach $z_{0}=0.765 \mathrm{M}$.

Since we use an expansion to finite order we cannot resolve the MOTS arbitrarily well. This means that, while we will find a minimum, it will typically be different from zero even for $z_{0}<0.765 M$. The value will, again, depend on a number of parameters, but primarily on the order of the expansion $L$. This can be seen very clearly in Fig. 3. In particular, for the lower-order expansions the expected drop at $z_{0}=0.765 \mathrm{M}$ cannot be detected at all, a significant decrease can only be seen for $L=8$. This demonstrates again that an early detection of a common MOTS will only be possible with a high-order expansion.

Also, this suggests that it is hardly possible to decide on the basis of the value of the sum $\mathcal{S}$ if a MOTS has been found. As a better test, we suggest checking whether $\Theta$ is 

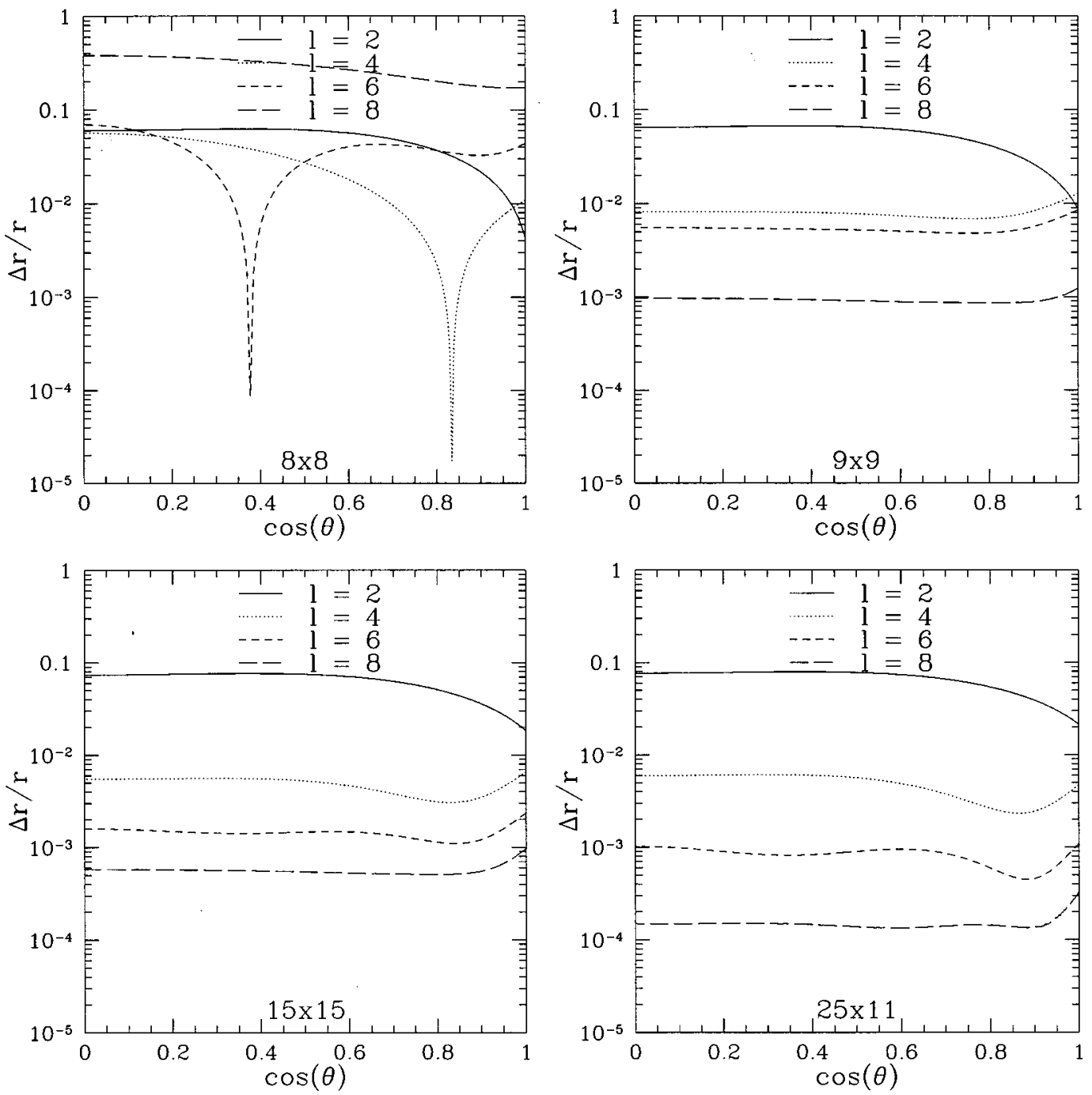

FIG. 2. Relative errors $\Delta r / r$ as a function of $\cos \theta$ for expansion to order $L=2,4,6$, and 8 and for different values of $N_{\Theta}=n_{\theta} \times n_{\phi}\left(z_{0}=0.6 M\right)$.

negative everywhere on a surface just inside the approximate MOTS. This surface "just inside" can be determined very easily by reducing the monopole term $F^{00}$ by a small fraction.

\section{SUMMARY}

We have developed an apparent-horizon finder based on a multipole expansion to arbitrary order $L$. The primary application we have in mind is the numerical evolution of a binary black-hole system. In order to check the performance of the MOTS finder in a spacetime of similar structure we have performed careful tests using initial data for two timesymmetric black holes.

From these tests it is evident that a reliable search for highly distorted MOTS requires high-order expansion. On the other hand, using a high-order expansion is very expensive and it is questionable if this will be affordable during a dynamical evolution.

However, in the evolution of a binary black-hole system, for example, it is desirable to detect a common MOTS as early as possible since the region interior to this surface no longer needs to be evolved. It may, therefore, be worthwhile searching for this common MOTS using a high-order expansion.

As a compromise, it is possible to use a low-order expansion for nearly spherical MOTS (as will be the case for the MOTS around individual black holes or the common MOTS in the later phases of an evolution) and a high-order expansion for highly distorted MOTS's (as in the early phase of the common MOTS). Unfortunately, "nearly spherical', is a coordinate-dependent concept in this context, and it is not clear how well the coordinates will behave in a binary blackhole evolution code.

An obvious optimization of the code is to change to a minimization scheme that uses derivatives. This is a fairly tedious, though straightforward, task.

\section{ACKNOWLEDGMENTS}

We would like to thank Peter Anninos and Edward Seidel for helpful discussions. This work was supported by NSF 


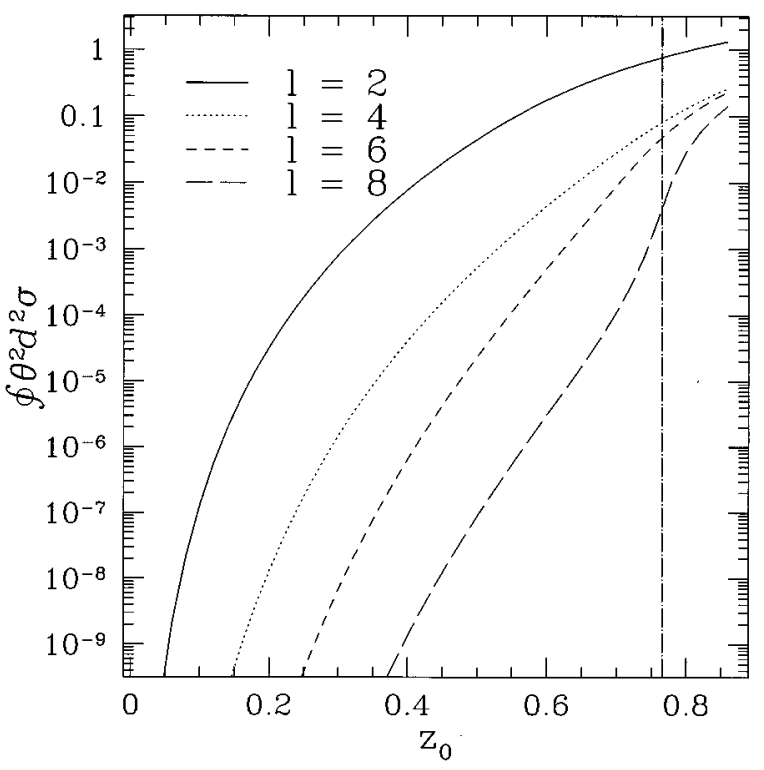

FIG. 3. Integrals over the expansion $\Theta$ [Eq. (18)] as a function of $z_{0}$ for expansions to order $L=2,4,6$, and 8 (using $N_{\Theta}=25 \times 11$ points). For $z_{0}<0.765 M$, i.e., left of the vertical line, the two black holes have an encompassing MOTS.

Grant Nos. AST 91-19475 and PHY 94-08378, NASA Grant No. NAG-2809, and by the Grand Challenge Grant Nos. NSF PHY 93-18152/ASC 93-18152 (ARPA supplemented). Computations were performed at the Cornell Center for Theory and Simulation in Science and Engineering, which is supported in part by the National Science Foundation, IBM Corporation, New York State, and the Cornell Research Institute.

\section{APPENDIX A: USEFUL EXPRESSIONS FOR STF TENSORS}

The purpose of this appendix is to provide several useful expressions for spherical harmonics and STF tensors that have been omitted in the main text. All these formulas are taken from Ref. [16].

The spherical harmonics can be written

$$
\begin{aligned}
Y^{l m}(\theta, \phi) & =C^{l m} e^{i m \phi} P^{l m}(\cos \theta) \text { for } 0 \leqslant m \leqslant l \\
& =C^{l m}\left(e^{i \phi} \sin \theta\right)^{m} \sum_{j=0}^{[(l-m) / 2]} a^{l m j}(\cos \theta)^{l-m-2 j} \\
& \text { for } 0 \leqslant m \leqslant l \\
& =(-1)^{m} Y^{l|m| *} \text { for }-l \leqslant m<0 .
\end{aligned}
$$

Here, * denotes complex conjugation, $[(l-m) / 2]$ means the largest integer less than or equal to $(l-m) / 2$, and

$$
\begin{aligned}
& C^{l m}=(-1)^{m}\left(\frac{2 l+1}{4 \pi} \frac{(l-m) !}{(l+m) !}\right)^{1 / 2}, \\
& a^{l m j}=\frac{(-1)^{j}}{2^{l} j !(l-j) !} \frac{(2 l-2 j) !}{(l-m-2 j) !} .
\end{aligned}
$$

Note that the Cartesian components of a radial unit vector $n$ can be written

$$
n_{x}+i n_{y}=e^{i \phi} \sin \theta, \quad n_{z}=\cos \theta .
$$

In terms of these, the spherical harmonics are

$$
Y^{l m}(\theta, \phi)=\mathcal{Y}_{K_{l}}^{l m} N_{K_{l}}(\theta, \phi),
$$

where the $\mathcal{Y}_{K_{l}}^{l m}$ are defined as

$$
\begin{aligned}
\mathcal{Y}_{K_{l}}^{l m}= & C^{l m} \sum_{j=0}^{[(l-m) / 2]} a^{l m j}\left(\delta_{\left(k_{1}\right.}^{1}+i \delta_{\left(k_{1}\right.}^{2}\right) \cdots\left(\delta_{\left(k_{m}\right.}^{1}+i \delta_{\left(k_{m}\right.}^{2}\right) \\
& \times \delta_{k_{m+1}}^{3} \cdots \delta_{k_{l-2 j}}^{3} \\
& \times\left(\delta_{k_{l-2 j+1}}^{a_{1}} \delta_{k_{l-2 j+2}}^{a_{1}}\right) \cdots\left(\delta_{k_{l-1}}^{a_{j}} \delta_{k_{l}}^{a_{j}}\right)
\end{aligned}
$$$$
\text { for } 0 \leqslant m \leqslant l
$$$$
=(-1)^{m}\left(\mathcal{Y}_{K_{l}}^{|| m \mid}\right)^{*} \text { for }-l \leqslant m<0 .
$$

$\delta_{k_{l}}^{a_{i}}$ is the Kronecker delta and the brackets $A_{\left(k_{1} \cdots k_{l}\right)}$ denote complete symmetrization. Obviously, the $\mathcal{Y}_{K_{l}}^{l m}$ are completely symmetric by definition, and they are also completely tracefree. Moreover, for every $l$ the $2 l+1$ different $\mathcal{Y}_{K_{l}}^{l m}$ are linearly independent and span a basis for the STF tensors of rank $l$. Any STF tensor of rank $l$ can, therefore, be written as

$$
\mathcal{F}_{K_{l}}=\sum_{m=-l}^{l} F^{l m} \mathcal{Y}_{K_{l}}^{l m}
$$

For $\mathcal{F}_{K_{l}}$ to be real, the coefficients have to satisfy $F^{l-m}=(-1)^{m}\left(F^{l m}\right)^{*}$.

\section{APPENDIX B: STORAGE}

Since we want to allow for expansion to arbitrary order $L$, we have to store objects of the type $N_{K_{l}}$ with multi-indices up to arbitrary length $L$. This can be accomplished by noting that all these objects are completely symmetric; i.e.,

$$
N_{k_{1} \cdots k_{i} \cdots k_{j} \cdots k_{l}}=N_{k_{1} \cdots k_{j} \cdots k_{i} \cdots k_{l}}
$$

for any pair of indices $k_{i}$ and $k_{j}$. In three dimensions these indices can only take the value $x, y$, or $z$. It is, therefore, sufficient to specify the number of indices with value $x, y$, and $z$ to determine any element in $N_{K_{l}}$ uniquely. In the following we adopt the notation

$$
N_{\left(N_{x}\right)\left(N_{y}\right)\left(N_{z}\right)},
$$

where the $N_{x}, N_{y}$, and $N_{z}$ are the total numbers of indices $x, y$, and $z$. Obviously, the rank of the tensor is

$$
l=N_{x}+N_{y}+N_{z}
$$

A symmetric tensor of rank $l$ has

$$
\sum_{i=0}^{l}(i+1)=\frac{1}{2}(l+1)(l+2)
$$


independent elements, the number of independent components for all symmetric tensors of rank up to $L$ is

$$
\sum_{l=0}^{L} \frac{1}{2}(l+1)(l+2)=\frac{1}{6}(L+1)(L+2)(L+3) .
$$

We can, therefore, store all symmetric tensors up to order $L$ in an array of this length. Each element is uniquely determined by a combination of $N_{x}, N_{y}$ and $N_{z}$.

Note that an element $N_{\left(N_{x}\right)\left(N_{y}\right)\left(N_{z}\right)}$ appears

$$
\frac{l !}{N_{x} ! N_{y} ! N_{z} !}
$$

times in a symmetric tensor. This weighting factor has to be taken into account when carrying out sums as in Eq. (12).

In our code we store $N_{K_{l}}$, its first and second derivatives, and the STF tensor $\mathcal{F}_{K_{l}}$ in arrays of this kind. In addition to being symmetric, the latter is completely trace-free, so that we could use even less storage. We decided not to do so, since this would complicate the code for little benefit.

The next objects that need to be stored are the basis STF tensors $\mathcal{Y}_{K_{l}}^{l m}$. For each $l$ this requires storage of $2 l+1$ symmetric tensors of rank $l$. We, therefore, need an array of length

$$
\begin{aligned}
\sum_{l=0}^{L} \frac{1}{2}(2 l+1)(l+1)(l+2) \\
\quad=\frac{1}{12}(3 L+2)(L+1)(L+2)(L+3) .
\end{aligned}
$$

Each element is uniquely labeled by a combination of $N_{x}$, $N_{y}$, and $N_{z}$ together with an index $m$.

As a further complication, these tensors are complex. However, since $\mathcal{Y}_{K_{l}}^{l(-m)}=(-1)^{m}\left(\mathcal{Y}_{K_{l}}^{l m}\right) *$ see Appendix A, Eq. (A6)], we need to store only the non-negative $m$. This can be accomplished by storing the real parts in the storage for $m \geqslant 0$ and the imaginary parts in the storage for $m<0$.

A sum as in Eq. (14) can be written

$$
\begin{aligned}
\mathcal{F}_{K_{l}} & =\sum_{m=-l}^{l} F^{l m} \mathcal{Y}_{K_{l}}^{l m} \\
& =F^{l 0} \mathcal{Y}_{K_{l}}^{l 0}+\sum_{m=1}^{l}\left[F^{l m} \mathcal{Y}_{K_{l}}^{l m}+\left(F^{l m} \mathcal{Y}_{K_{l}}^{l m}\right)^{*}\right] \\
& =F^{l 0} \mathcal{Y}_{K_{l}}^{l 0}+2 \sum_{m=1}^{l}\left[\mathfrak{R} F^{l m} \mathfrak{R} \mathcal{Y}_{K_{l}}^{l m}-\mathfrak{I} F^{l m} \mathfrak{I} \mathcal{Y}_{K_{l}}^{l m}\right]
\end{aligned}
$$

[where we have assumed $F^{l-m}=(-1)^{m}\left(F^{l m}\right)^{*}$ so that $\mathcal{F}_{K_{l}}$ is real, see Appendix A]. Storing the elements as described above, we can again sum from $-l$ to $l$, but we have to take into account a weight of 2 for $m>0$ and -2 for $m<0$, as given by Eq. (B8).

The last objects that have to be stored are the expansion coefficients $F^{l m}$. Since for each $l$ there are $2 l+1$ different elements, we have to store a total of

$$
\sum_{l=0}^{L}(2 l+1)=(L+1)^{2}
$$

elements. Again, these elements will be complex and satisfy $F^{l-m}=(-1)^{m}\left(F^{l m}\right)^{*}$, so that we can again store the real parts in the storage for $m \geqslant 0$ and the imaginary parts in the storage for $m<0$.

\section{APPENDIX C: INITIALIZATION AND RECURRENCE RELATIONS}

Before a MOTS can be found, $N_{K_{l}}$, its derivatives, and $\mathcal{Y}_{K_{l}}^{l m}$ need to be initialized for various directions $(\theta, \phi)$. Although direct expressions could be used to do so [as for example Eq. (A6) in Appendix A], this would be extremely tedious and inefficient. Moreover, sums such as Eq. (A6) are prone to a cancellation error from adding terms of opposite sign. We have, therefore, derived recursive expressions that make the initialization much easier.

Since the $\mathcal{Y}_{K_{l}}^{l m}$ are closely related to the spherical harmonics, we can start with the standard recurrence relation

$$
\begin{aligned}
Y^{l m}= & \sqrt{\frac{2 l+1}{l^{2}-m^{2}}}\left(\sqrt{2 l-1} Y^{(l-1) m} \cos \theta\right. \\
& \left.-\sqrt{\frac{(l-1)^{2}-m^{2}}{2 l-3}} Y^{(l-2) m}\right) .
\end{aligned}
$$

Here, we can use $\cos \theta=n_{z}$, insert $n_{x}^{2}+n_{y}^{2}+n_{z}^{2}=1$, and replace the spherical harmonics with Eq. (15), which yields

$$
\begin{aligned}
\mathcal{Y}_{K_{l}}^{l m} N_{K_{l}}= & \sqrt{\frac{2 l+1}{l^{2}-m^{2}}}\left(\sqrt{2 l-1} \mathcal{Y}_{K_{l-1}}^{(l-1) m} N_{K_{l-1}} n_{z}\right. \\
& -\sqrt{\frac{(l-1)^{2}-m^{2}}{2 l-3}} \mathcal{Y}_{K_{l-2}^{(l-2) m}}^{(l-3} N_{K_{l-2}} \\
& \left.\times\left(n_{x}^{2}+n_{y}^{2}+n_{z}^{2}\right)\right) .
\end{aligned}
$$

Adopting our notation (B2), we can rewrite this equation as

$$
\begin{aligned}
& \frac{l !}{N_{x} ! N_{y} ! N_{z} !} \mathcal{Y}_{\left(N_{x}\right)\left(N_{y}\right)\left(N_{z}\right)}^{l m} N_{\left(N_{x}\right)\left(N_{y}\right)\left(N_{z}\right)} \\
& =\sqrt{\frac{2 l+1}{l^{2}-m^{2}}\left[\frac{(l-1) !}{N_{x} ! N_{y} !\left(N_{z}-1\right) !} \sqrt{2 l-1} \mathcal{Y}_{\left(N_{x}\right)\left(N_{y}\right)\left(N_{z}-1\right)}^{(l-1)}\right.} \\
& \quad-\sqrt{\frac{(l-1)^{2}-m^{2}}{2 l-3}}\left(\frac{(l-2) !}{\left(N_{x}-2\right) ! N_{y} ! N_{z} !} \mathcal{Y}_{\left(N_{x}-2\right)\left(N_{y}\right)\left(N_{z}\right)}^{(l-2) m}\right. \\
& \quad+\frac{(l-2) !}{N_{x} !\left(N_{y}-2\right) ! N_{z} !} \mathcal{Y}_{\left(N_{x}\right)\left(N_{y}-2\right)\left(N_{z}\right)}^{(l-2) m} \\
& \left.\left.\quad+\frac{(l-2) !}{N_{x} ! N_{y} !\left(N_{z}-2\right) !} \mathcal{Y}_{\left(N_{x}\right)\left(N_{y}\right)\left(N_{z}-2\right)}^{(l-2) m}\right)\right] N_{\left(N_{x}\right)\left(N_{y}\right)\left(N_{z}\right)}
\end{aligned}
$$


Note that, because the $\mathcal{Y}_{N_{K_{l}}}^{l m}$ are direction independent, only specific terms in the STF tensor sums are allowed on the right-hand side of Eq. (C2). Only those terms that, together with the additional unit vectors appearing on the right-hand side of Eq. (C2), result in the same number of $N_{x}, N_{y}$, and $N_{z}$ that appear on its left-hand side are allowed. Finally, the direction independence of the $\mathcal{Y}_{N_{K_{l}}}^{l m}$ means that we can drop the $N_{\left(N_{x}\right)\left(N_{y}\right)\left(N_{z}\right)}$ terms (which contain all of the directional dependence) to get

$$
\begin{aligned}
\mathcal{Y}_{\left(N_{x}\right)\left(N_{y}\right)\left(N_{z}\right)}^{l m}= & \sqrt{\frac{2 l+1}{l^{2}-m^{2}}}\left[\frac{N_{z}}{l} \sqrt{2 l-1} \mathcal{Y}_{\left(N_{x}\right)\left(N_{y}\right)\left(N_{z}-1\right)}^{(l-1) m}\right. \\
& -\frac{1}{l(l-1)} \sqrt{\frac{(l-1)^{2}-m^{2}}{2 l-3}} \\
& \times\left[N_{x}\left(N_{x}-1\right) \mathcal{Y}_{\left(N_{x}-2\right)\left(N_{y}\right)\left(N_{z}\right)}^{(l-2) m}\right. \\
& +N_{y}\left(N_{y}-1\right) \mathcal{Y}_{\left(N_{x}\right)\left(N_{y}-2\right)\left(N_{z}\right)}^{(l-2) m} \\
& \left.+N_{z}\left(N_{z}-1\right) \mathcal{Y}_{\left(N_{x}\right)\left(N_{y}\right)\left(N_{z}-2\right)}^{(l-2) m}\right] .
\end{aligned}
$$

This expression can be used to initialize the $\mathcal{Y}_{K_{l}}^{l m}$ with $-l<m<l$. For $m=l$, we can use Eq. (A6), which, for this case, reduces to

$$
\mathcal{Y}_{\left(N_{x}\right)\left(N_{y}\right)\left(N_{z}\right)}^{l l}=\left\{\begin{array}{l}
0 \quad \text { if } N_{z} \neq 0, \\
\frac{(-1)^{l} i^{\left(N_{y}\right)}}{2^{l} l !} \sqrt{\frac{(2 l+1)(2 l) !}{4 \pi}} \text { if } N_{z}=0 .
\end{array}\right.
$$

Next, we have to initialize the tensors $N_{K_{l}}$ [Eq. (13)] as well as their first and second derivatives. Note that the partial derivative of a unit vector is

$$
\partial_{i} n_{j}=\frac{1}{r}\left(\delta_{i j}-n_{i} n_{j}\right) \equiv n_{i j} .
$$

The partial derivative of $N_{K_{l}}$ is, therefore,

$$
\partial_{i} N_{K_{l}}=\partial_{i}\left(n_{k_{1}} n_{k_{2}} \cdots n_{k_{l}}\right)=\ln _{i\left(k_{l}\right.} N_{K_{l-1)}} .
$$

Taking a second derivative yields, after some algebra,

$$
\begin{aligned}
\partial_{i} \partial_{j} N_{K_{l}}= & -\frac{l}{r}\left(n_{i j} N_{K_{l}}+n_{i} n_{j\left(k_{l}\right.} N_{K_{l-1)}}+n_{j} n_{i\left(k_{l}\right.} N_{\left.K_{l-1}\right)}\right) \\
& +l(l-1) n_{i\left(k_{l-1}\right.} N_{K_{l-2}} n_{\left.k_{l}\right)^{j}} .
\end{aligned}
$$

Note that we can now construct $N_{K_{l}}, \partial_{i} N_{K_{l}}$, and $\partial_{i} \partial_{j} N_{K_{l}}$ from the ten different, totally symmetric objects $N_{K_{l}}$, $n_{i\left(k_{l}\right.} N_{\left.K_{l-1}\right)}$, and $n_{i\left(k_{l-1}\right.} N_{K_{l-2}} n_{\left.k_{l}\right)^{j}}$. Denoting any one of these with $S_{\left(N_{x}\right)\left(N_{y}\right)\left(N_{z}\right)}$ [in our notation (B2)], we find that they all satisfy the recurrence relation

$$
S_{\left(N_{x}\right)\left(N_{y}\right)\left(N_{z}\right)}=\frac{N_{x} n_{x} S_{\left(N_{x}-1\right)\left(N_{y}\right)\left(N_{z}\right)}+N_{y} n_{y} S_{\left(N_{x}\right)\left(N_{y}-1\right)\left(N_{z}\right)}+N_{z} n_{z} S_{\left(N_{x}\right)\left(N_{y}\right)\left(N_{z}-1\right)}}{N_{x}+N_{y}+N_{z}} .
$$

The starting values for the different objects are

$$
\begin{array}{cccc} 
& N_{K_{l}} & n_{a\left(k_{l}\right.} N_{\left.K_{l-1}\right)} & n_{a\left(k_{l-1}\right.} N_{K_{l-2}} n_{\left.k_{l}\right) b} \\
l=0 & 1 & 0 & 0 \\
l=1 & n_{i} & n_{a i} & 0 \\
l=2 & n_{i} n_{j} & \frac{1}{2}\left(n_{a i} n_{j}+n_{a j} n_{i}\right) & \frac{1}{2}\left(n_{a i} n_{b j}+n_{a j} n_{b i}\right) .
\end{array}
$$

Once these objects have been calculated up to order $L$, we can then construct the derivatives of $N_{K_{l}}$ using Eqs. (C7) and (C8).

\section{APPENDIX D: THE AREA ELEMENT ON THE APPARENT HORIZON}

Beginning with the line element for the three-dimensional spatial hypersurface

$$
d s^{2}=g_{i j} d x^{i} d x^{j}
$$

we transform to polar coordinates centered around some point using

$$
d x^{i}=\frac{\partial x^{i}}{\partial r} d r+\frac{\partial x^{i}}{\partial \theta} d \theta+\frac{\partial x^{i}}{\partial \phi} d \phi
$$

The line element for the surface parametrized by $r=f(\theta, \phi)$ is obtained by substituting

$$
d r=\frac{\partial f}{\partial \theta} d \theta+\frac{\partial f}{\partial \phi} d \phi
$$

into Eq. (D2). We define

$$
\Theta^{i}=\frac{1}{r}\left(\frac{\partial x^{i}}{\partial r} \frac{\partial f}{\partial \theta}+\frac{\partial x^{i}}{\partial \theta}\right)
$$

and

$$
\Phi^{i}=\frac{1}{r \sin \theta}\left(\frac{\partial x^{i}}{\partial r} \frac{\partial f}{\partial \phi}+\frac{\partial x^{i}}{\partial \phi}\right)
$$

which can be expanded to yield

$\Theta^{x}=\frac{n_{x} n_{z}}{\sqrt{1-n_{z}^{2}}}\left(1+n_{x} \partial_{x} f+n_{y} \partial_{y} f\right)-n_{x} \sqrt{1-n_{z}^{2}} \partial_{z} f$ 


$$
\begin{array}{r}
\Theta^{y}=\frac{n_{y} n_{z}}{\sqrt{1-n_{z}^{2}}}\left(1+n_{x} \partial_{x} f+n_{y} \partial_{y} f\right)-n_{y} \sqrt{1-n_{z}^{2}} \partial_{z} f, \\
\Theta^{z}=\frac{n_{z}^{2}}{\sqrt{1-n_{z}^{2}}}\left(n_{x} \partial_{x} f+n_{y} \partial_{y} f\right)-\sqrt{1-n_{z}^{2}}\left(1+n_{z} \partial_{z} f\right), \\
\Phi^{x}=\frac{1}{\sqrt{1-n_{z}^{2}}}\left[n_{x}\left(n_{x} \partial_{y} f-n_{y} \partial_{x} f\right)-n_{y}\right], \\
\Phi^{y}=\frac{1}{\sqrt{1-n_{z}^{2}}}\left[n_{y}\left(n_{x} \partial_{y} f-n_{y} \partial_{x} f\right)+n_{x}\right],
\end{array}
$$

$$
\Phi^{z}=\frac{1}{\sqrt{1-n_{z}^{2}}}\left(n_{x} \partial_{y} f-n_{y} \partial_{x} f\right) .
$$

Finally, we can now rewrite the line element (D1) as

$$
d s^{2}=f^{2}\left(\Theta^{i} \Theta_{i} d \theta^{2}+2 \Theta^{i} \Phi_{i} \sin \theta d \theta d \phi+\Phi^{i} \Phi_{i} \sin ^{2} \theta d \phi^{2}\right)
$$

where we have lowered the indices on $\Theta$ and $\Phi$ with the metric $g_{i j}$. From this we find the two-surface area element

$d^{2} \sigma=\sqrt{\left(\Theta^{i} \Theta_{i}\right)\left(\Phi^{j} \Phi_{j}\right)-\left(\Theta^{k} \Phi_{k}\right)^{2}} f^{2} \sin \theta d \theta d \phi$.

(D13)
[1] E. Seidel and W.-M. Suen, Phys. Rev. Lett. 69, 1845 (1992).

[2] S. W. Hawking and G. F. R. Ellis, The Large Scale Structure of Spacetime (Cambridge University Press, Cambridge, England, 1973).

[3] A. Čadež, Ann. Phys. (N.Y.) 83, 449 (1974).

[4] N. T. Bishop, Gen. Relativ. Gravit. 14, 717 (1982).

[5] N. T. Bishop, Gen. Relativ. Gravit. 16, 589 (1984).

[6] S. L. Shapiro and S. A. Teukolsky, Phys. Rev. D 45, 2739 (1992).

[7] K. Eppley, Phys. Rev. D 16, 1609 (1977).

[8] G. B. Cook and J. W. York, Jr., Phys. Rev. D 41, 1077 (1990).

[9] G. B. Cook and A. M. Abrahams, Phys. Rev. D 46, 702 (1992).

[10] K. P. Tod, Class. Quantum Grav. 8, L115 (1991).

[11] T. Nakamura, Y. Kojima, and K. Oohara, Phys. Lett. 106A, 235 (1984).
[12] T. Nakamura, Y. Kojima, and K. Oohara, Phys. Lett. 107A, 452 (1985).

[13] A. J. Kemball and N. T. Bishop, Class. Quantum Grav. 8, 1361 (1991).

[14] M. F. Huq, S. A. Klasky, M. W. Choptuik, and R. A. Matzner (unpublished).

[15] J. Libson, J. Massó, E. Seidel, and W.-M. Suen, in General Relativity, Proceedings of the 7th Marcel Grossmann Meeting, Stanford, California, 1994, edited by R. Ruffini and M. Keiser (World Scientific, Singapore, 1995), Report No. gr-qc/ 9412058 (unpublished).

[16] K. S. Thorne, Rev. Mod. Phys. 52, 299 (1980).

[17] W. H. Press, S. A. Teukolsky, W. T. Wetterling, and B. P. Flannery, Numerical Recipes in $C$, 2nd ed. (Cambridge University Press, Cambridge, England, 1992). 